\title{
Inflammation and Nocturnal Pattern of Blood Pressure in Normotensives
}

\author{
Seyda Gunay, ${ }^{10}$ Serhat Çalışkan, ${ }^{\text {(D) }}$ Deniz Sigirli ${ }^{10}$ \\ Bursa Uludag University, ${ }^{\prime}$ Bursa - Turkey \\ Istanbul Bahçelievler State Hospital, Instanbul - Turkey
}

\section{Abstract}

Background: In most healthy individuals, blood pressure (BP) shows a circadian rhythm. Being non-dipper increases cardiovascular risk in normotensive and hypertensive individuals. Nocturnal dipping shows a correlation with the state of inflammation.

Objetive: To investigate the relationship between inflammation-based indexes and nocturnal BP pattern in normotensive individuals.

Method: This is a retrospective study that included patients evaluated with ambulatory BP monitoring (ABPM). A total of 131 normotensive individuals were included and grouped as dippers and non-dippers. The normality of the data was verified with a Shapiro-Wilk test. We compared ABPM variables and inflammation-based indexes derived from blood tests (monocyte to high-density lipoprotein ratio [MHR], platelet to lymphocyte ratio [PLR], neutrophil to lymphocyte ratio [NLR], and systemic immune-inflammation index [SII]) between groups. The independent samples t-test and Mann-Whitney $U$ test were used for comparing variables with normal and nonnormal distributions, respectively. The Pearson's chi-squared test was used to compare categorical variables, and Spearman's correlation coefficient was used to examine the relationships between variables. A receiver operating characteristic (ROC) curve was used to evaluate the diagnostic performances of inflammation-based indexes. The level of statistical significance was $5 \%$.

Results: The study included 131 patients (mean \pm standard deviation [SD] age 49.2 \pm 15.1 years, 58 [76.0\%] of which were women). SII was significantly higher in the non-dipper group ( $p=0.033)$. Significant negative correlations were observed between the change in systolic BP $[\triangle \mathrm{SBP}]$ and SII $(\mathrm{r}=-0.172, \mathrm{p}=0.049)$ and between $\triangle$ SBP and PLR $(\mathrm{r}=-0.179, \mathrm{p}=0.040)$.

Conclusion: SII is a predictor of nocturnal BP pattern in normotensives.

Keywords: Inflammation; Monocytes; HDL Cholesterol; Hypertension.

\section{Introduction}

Blood pressure (BP) normally decreases during sleep, and certain metabolic and cardiovascular alterations may affect this circadian pattern. ${ }^{1}$ Leading reasons for a non-dipping BP pattern are obesity, sleep disorders, obstructive sleep apnea, chronic kidney disease, excessive salt consumption, diabetes mellitus, orthostatic hypotension, autonomic dysfunction, and advanced age. ${ }^{2}$ An arbitrary cut-off point has been proposed to define patients as "dippers" if their nocturnal BP falls by $\geq 10 \%$ of the daytime mean BP value. ${ }^{2} \mathrm{~A}$ non-dipper BP pattern is associated with high cardiovascular mortality and morbidity in people with both normal BP and hypertension. ${ }^{3,4}$

In recent years, hematological parameters such as the neutrophil to lymphocyte ratio (NLR), platelet to lymphocyte ratio (PLR), monocyte to high-density lipoprotein (HDL) cholesterol ratio (MHR), and systemic immune-inflammation index (SII) have been investigated in different systemic diseases as indicators of inflammation..$^{5-12}$ Although studies are searching for

Mailing Address: Seyda Gunay

Görükle Kampusu. Postal Code: 16059, Bursa - Turkey

E-mail: seydagunaymedical@yahoo.com 
the relationship between inflammation and nocturnal BP pattern in hypertensive patients, ${ }^{13,14}$ to the best of our knowledge, these easily accessible parameters have not been used in studies with normotensive individuals. Hence, in this study we aimed to investigate the relationship between inflammation-based indexes and nocturnal BP pattern in normotensive individuals.

\section{Materials and Methods}

\section{Study Population}

Patients admitted to the outpatient cardiology clinic at Hospital were retrospectively screened. Those who were evaluated with ambulatory BP monitoring (ABPM) to confirm/reject a hypertension diagnosis were enrolled. Our sample represented patients aged 18 years or older, without a history of hypertension or treatment with antihypertensives, and with ABPM findings compatible with normotension in consistence with the European Society of Hypertension/European Society of Cardiology (ESH/ESC) guidelines ${ }^{2}$ (daytime threshold for hypertension: systolic $\mathrm{BP}[\mathrm{SBP}] \geq 135 \mathrm{mmHg}$ and/or diastolic $\mathrm{BP}[\mathrm{DBP}] \geq 85$ $\mathrm{mmHg}$; nighttime: $\mathrm{SBP} \geq 120 \mathrm{mmHg}$ and/or $\mathrm{DBP} \geq 70$ $\mathrm{mmHg}$; overall 24-hour mean: $\mathrm{SBP} \geq 130 \mathrm{mmHg}$ and/ or $\mathrm{DBP} \geq 80 \mathrm{mmHg}$ ). Exclusion criteria were pregnancy; history of drug/alcohol abuse; having a night shift job; chronic inflammatory disease; kidney failure; thyroid function disorders; connective tissue disease; chronic liver disease; acute infection; sleep disorders; malignancy; use of antinflammatory drugs, statins, or drugs that may increase $\mathrm{BP}$ (such as steroids); and intolerance to ABPM. The sample size was defined for convenience. A total of 131 patients were included. Participants represented a consecutive series of patients fulfilling these exclusion/inclusion criteria and were divided into 2 groups (dippers vs non-dippers) according to the decline in nighttime SBP. The dipper group was defined as patients with a nocturnal dip of $\geq 10 \%$ in SBP. The non-dipper group was defined as patients with a nocturnal dip of $<10 \%$ in SBP. ${ }^{2}$

This study was approved by the local ethics committee of Istanbul Bakirkoy Dr Sadi Konuk Training and Research Hospital (20.05.2019, 2019-10-10).

\section{ABPM Assessment}

A portable recording device (Suntech Bravo 24-HR ABP) was used to record 24-h ABPM values. The cuff was placed on the patient's non-dominant arm. Overall, the nighttime and daytime SBP and DBP of each participant were automatically measured every $20 \mathrm{~min}$ between 07:00 and 23:00 $\mathrm{h}$ and every $30 \mathrm{~min}$ during the night. Daytime and nighttime periods were defined using fixed time periods for all patients. Participants were asked to continue performing their usual activities. Data was analyzed using SunTech AccuWin Pro v3 ABPM software. Mean SBP and DBP values, as well as mean arterial pressure (MAP), were separetely calculated for nighttime and daytime periods. BP series were excluded if $\leq 70 \%$ of the measurements were valid. The percentage of nocturnal BP decline was calculated using the following formula: nocturnal BP decline $(\%)=$ (daytime BP- nocturnal BP)×100 / daytime BP.

\section{Study Parameters}

Data regarding patient demographics, blood biochemistry (total cholesterol, triglycerides, low-density lipoprotein cholesterol, HDL), complete blood count at admission, and ABPM results were obtained from the medical records. The 24-hour, nighttime, and daytime ABPM values of SBP, DBP and MAP, as well as the mean nocturnal declines in SBP, DBP, and MAP, were recorded for all patients.

SII (calculated as platelet count $x$ neutrophil count / lymphocyte count), NLR, PLR, and MHR were constructed as inflammation-based indexes in accordance with previous studies. ${ }^{9-12,15,16}$

\section{Statistical Analysis}

Our statistical analysis was performed using IBM SPSS Statistics version 21 and MedCalc version 12.3.0.0. The normality of the data was tested with the Shapiro-Wilk test. Normally distributed variables were presented as means \pm standard deviations (SDs); non-normally distributed variables were reported as median (interquartile range [IQR]) values. Independent samples t-tests and Mann-Whitney $U$ tests were used for comparing normal and non-normal variables, respectively, between the 2 indepedent groups. Categorical variables were presented as frequencies and percentages (n, \%), and Pearson's chi-squared tests were used to compare categorical variables between groups. The Spearman's correlation coefficient was used to examine the relationships between variables. A receiver operating characteristic (ROC) curve analysis was performed to evaluate the diagnostic performances of inflammation-based 
indexes. The Youden's J index was used to obtain the optimal cut-off value. The level of statistical significance adopted for our analyses was 5\%.

\section{Results}

This single-center, retrospective study included 131 patients (mean \pm SD age $49.20 \pm 15.09$ years, 58 (76.0\%) of which were women). Among these 131 patients, 55 (42.0\%) were included in the dipper group and $76(58.0 \%)$, in the non-dipper group. Baseline charecteristics and laboratory findings of the groups are summarized on Table-1. No significant differences were noticed between groups in terms of patient characteristics.

ABPM results of both groups were summarized on Figures-1 and -2. A significant difference was observed between dippers and non-dippers in terms of daytime and nighttime SBP and DBP. The difference between dippers and non-dippers in terms of daytime SBP was (mean \pm SD) $121.24 \pm 7.43$ vs $114.25 \pm 7.46$, respectively, $\mathrm{p}<0.001$, while for daytime DBP it was $($ mean $\pm S D)$ $73.20 \pm 6.11$ vs $68.46 \pm 5.68, p<0.001$. The difference between dippers and non-dippers in terms of nighttime SBP was (median [IQR]) 102 (15.0) vs 109 (9.8), respectively, $\mathrm{p}<0.001$, and in terms of nighttime DBP it was (median [IQR]) 60.0 (8.0) vs 63 (5.8), $\mathrm{p}<0.001$. The difference between dippers and non-dippers in terms of daytime MAP was (median [IQR]) 90.0 (9.33) vs 84.0 (9.08), respectively, $\mathrm{p}<0.001$, and that for nighttime MAP was (median [IQR]) 73.33 (9.0) vs 78.5 (6.5), $\mathrm{p}<0.001$; these were also statistically significant.

MHR, PLR, and NLR were similar between dippers and non-dippers $(\mathrm{p}=0.929, \mathrm{p}=0.110$, and $\mathrm{p}=0.152$, respectively). However, SII was significantly higher in the non-dipper group than in the dipper group (median [IQR]): 457.4 (233.5) vs 391.4 (266.6), respectively, $\mathrm{p}=0.033$ (Table-2). When we investigated the correlations between the change in systolic BP $(\triangle S B P)$ and inflammation-based indexes, we found significant negative correlations between $\triangle \mathrm{SBP}$ and SII ( $\mathrm{r}=-0.172$, $\mathrm{p}=0.049)$ and between $\triangle \mathrm{SBP}$ and PLR $(\mathrm{r}=-0.179, \mathrm{p}=0.040)$ in non-dipper normotensives. On the other hand, correlations between $\triangle S B P$ and MHR $(p=0.768)$ and between $\triangle$ SBP and NLR ( $p=0.320)$ were not significant in non-dipper normotensives.

Table 1 - Baseline charecteristics and laboratory findings of dipper and non-dipper groups

\begin{tabular}{|c|c|c|c|}
\hline & $\begin{array}{l}\text { Dipper } \\
(n=55)\end{array}$ & $\begin{array}{l}\text { Non-dipper } \\
\quad(n=76)\end{array}$ & p-value \\
\hline Age (years) ${ }^{*}$ & $49.7 \pm 14.1$ & $48.8 \pm 15.9$ & 0.743 \\
\hline Gender (female) \&x & $40(41.7)$ & $56(58.3)$ & 1.000 \\
\hline Total cholesterol (mg/dL) & $202.1 \pm 42.1$ & $206.1 \pm 37.6$ & 0.570 \\
\hline $\mathrm{TG}(\mathrm{mg} / \mathrm{dL})^{\sharp}$ & $133(103)$ & $105(82.3)$ & 0.408 \\
\hline $\mathrm{HDL}(\mathrm{mg} / \mathrm{dL})^{\#}$ & $50(16)$ & $51(17.8)$ & 0.879 \\
\hline $\mathrm{LDL}(\mathrm{mg} / \mathrm{dL})^{*}$ & $123.7 \pm 36.7$ & $128.7 \pm 29.7$ & 0.390 \\
\hline $\mathrm{WBC}\left(\times 10^{9} / \mathrm{L}\right)^{\#}$ & $7.59(3.29)$ & $7.45(2.45)$ & 0.928 \\
\hline Neutrophil $\left(\times 10^{9} / \mathrm{L}\right)^{\#}$ & $406(186)$ & $419.50(204.3)$ & 0.509 \\
\hline Monocyte $\left(x 10^{9} / \mathrm{L}\right)^{\#}$ & $61(39)$ & $59(27)$ & 0.946 \\
\hline Lymphocyte $\left(\times 10^{9} / \mathrm{L}\right)^{\#}$ & $244(131)$ & $241.50(84.3)$ & 0.475 \\
\hline RDW \%\# & $13.40(1.3)$ & $13.50(1.65)$ & 0.931 \\
\hline $\operatorname{PLT}\left(x 10^{9} / \mathrm{L}\right)^{*}$ & $256.6 \pm 62.9$ & $269.7 \pm 58.3$ & 0.223 \\
\hline $\mathrm{MPV}^{\sharp}(\mathrm{fL})$ & $10.20(1.3)$ & $10.25(1.18)$ & 0.814 \\
\hline
\end{tabular}



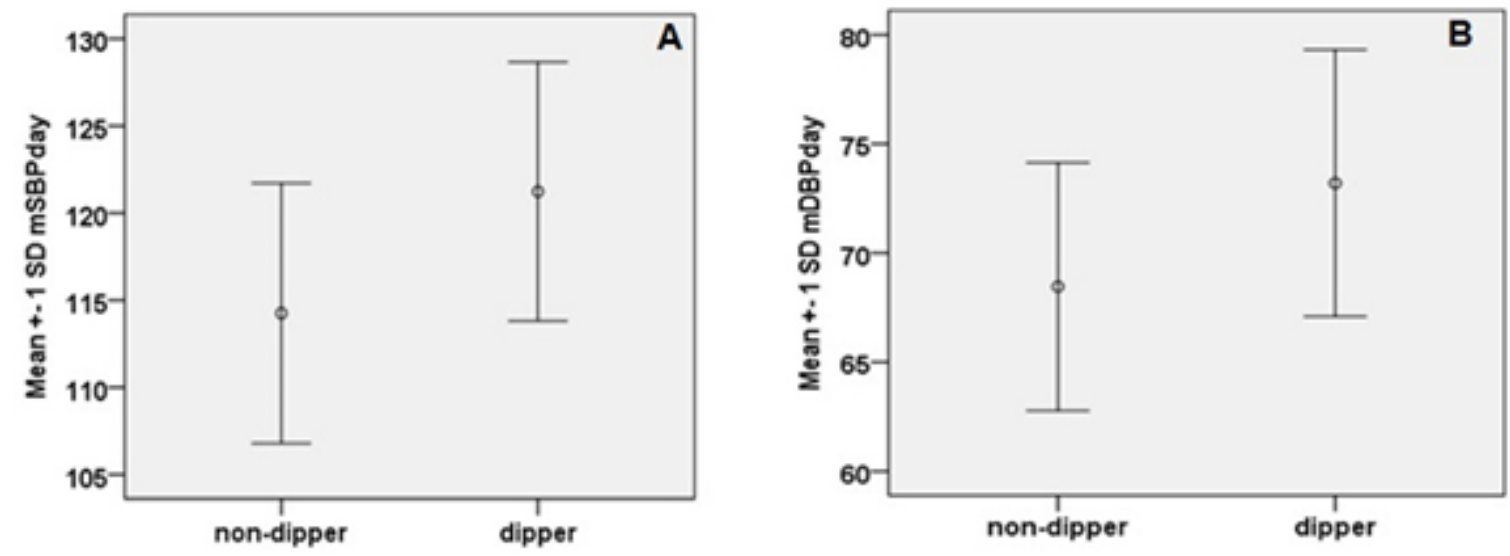

Figure 1 - Difference between dippers and non-dippers in terms of daytime blood pressure. A) Systolic blood pressure; B) Diastolic blood pressure (mSBP: mean systolic blood pressure; mDBP: mean diastolic blood pressure; SD: standard deviation). Error bars represent $1 \mathrm{SD}$, data present mean $\pm \mathrm{SD}$ values.
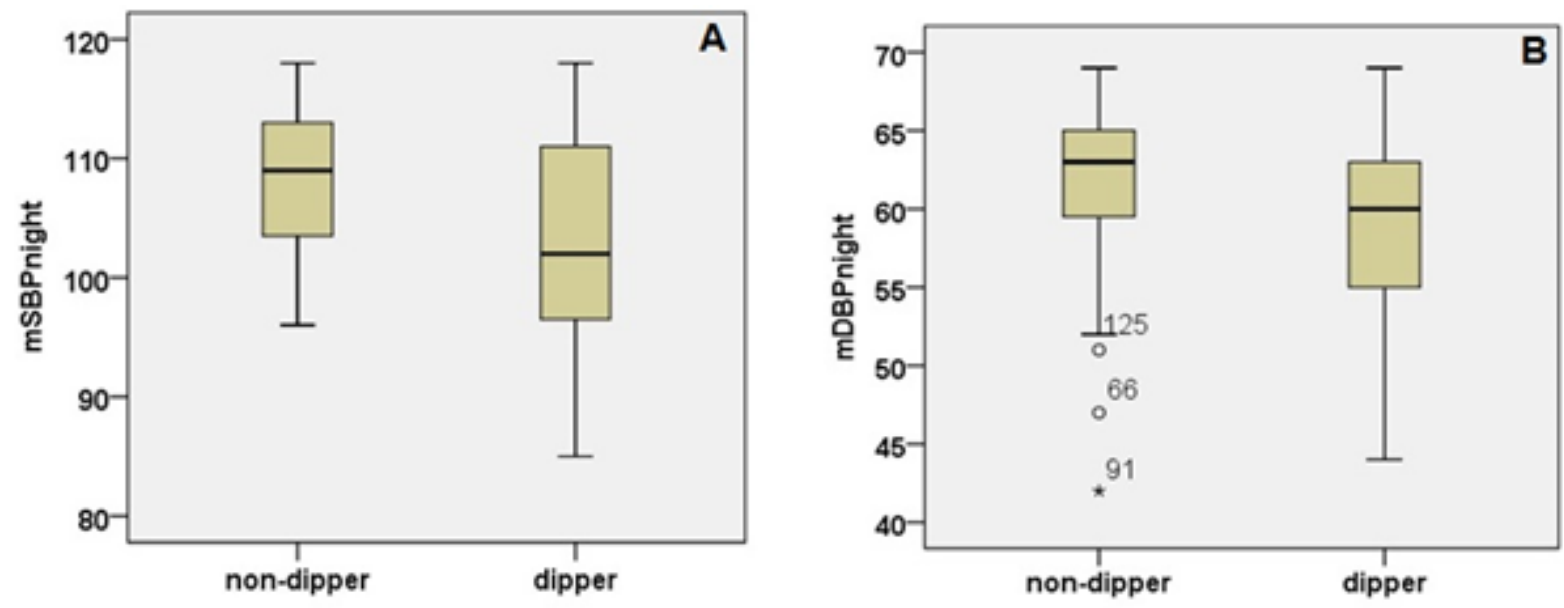

Figure 2 - Difference between dippers and non-dippers in terms of nighttime blood pressure A) Systolic blood pressure B) Diastolic blood pressure (mSBP: median systolic blood pressure; mDBP: median diastolic blood pressure; IQR: interquartile range). $\stackrel{\circ}{\text { : outliers; }}$ *: extreme values. Data presented as median [IQR] values.

In our ROC curve analyses, we found a significant diagnostic performance for SII in discriminating between dippers and non-dippers (area under the ROC curve [AUC ] $=0.610, p=0.031$ ). The optimal cut-off point for non-dippers was $>373.23$, corresponding to a $78.95 \%$ (95\% confidence interval [CI]: 68.1-87.5) sensitivity and a 49.09\% (95\% CI: 35.4-62.9) specificity. AUCs for MHR $(\mathrm{p}=0.932)$, PLR $(\mathrm{p}=0.118)$, and NLR $(\mathrm{p}=0.149)$ were not statistically significant (Figure-3).

\section{Discussion}

In this study, we found that among the studied parameters, only SII differed significantly between groups. Moreover, SII and PLR were negatively correlated with a nocturnal decline of SBP in non-dipper normotensives. MHR, NLR, and PLR values were similar between dipper and non-dipper normotensives. 
Table 2 - Inflammation-based indexes derived from laboratory tests

\begin{tabular}{lccc}
\hline & $\begin{array}{c}\text { Dipper } \\
(\mathbf{n}=55)\end{array}$ & $\begin{array}{c}\text { Non-dipper } \\
(\mathbf{n}=\mathbf{7 6})\end{array}$ & p-value \\
\hline SII & $391.4(266.6)$ & $457.4(233.5)$ & 0.033 \\
\hline MHR & $1.18(1.0)$ & $1.21(0.71)$ & 0.929 \\
\hline PLR & $0.96(0.44)$ & $1.09(0.34)$ & 0.110 \\
\hline NLR & $1.68(0.86)$ & $1.89(1.0)$ & 0.152 \\
\hline $\begin{array}{l}\text { Data presented as median (interquartile range [IQR]) values. } \\
\text { MHR: monocyte to high-density lipoprotein ratio; NLR: neutrophil to lymphocyte ratio; PLR: platelet to lymphocyte ratio; SII: systemic immune- } \\
\text { inflammation index. }\end{array}$ & \\
\hline
\end{tabular}

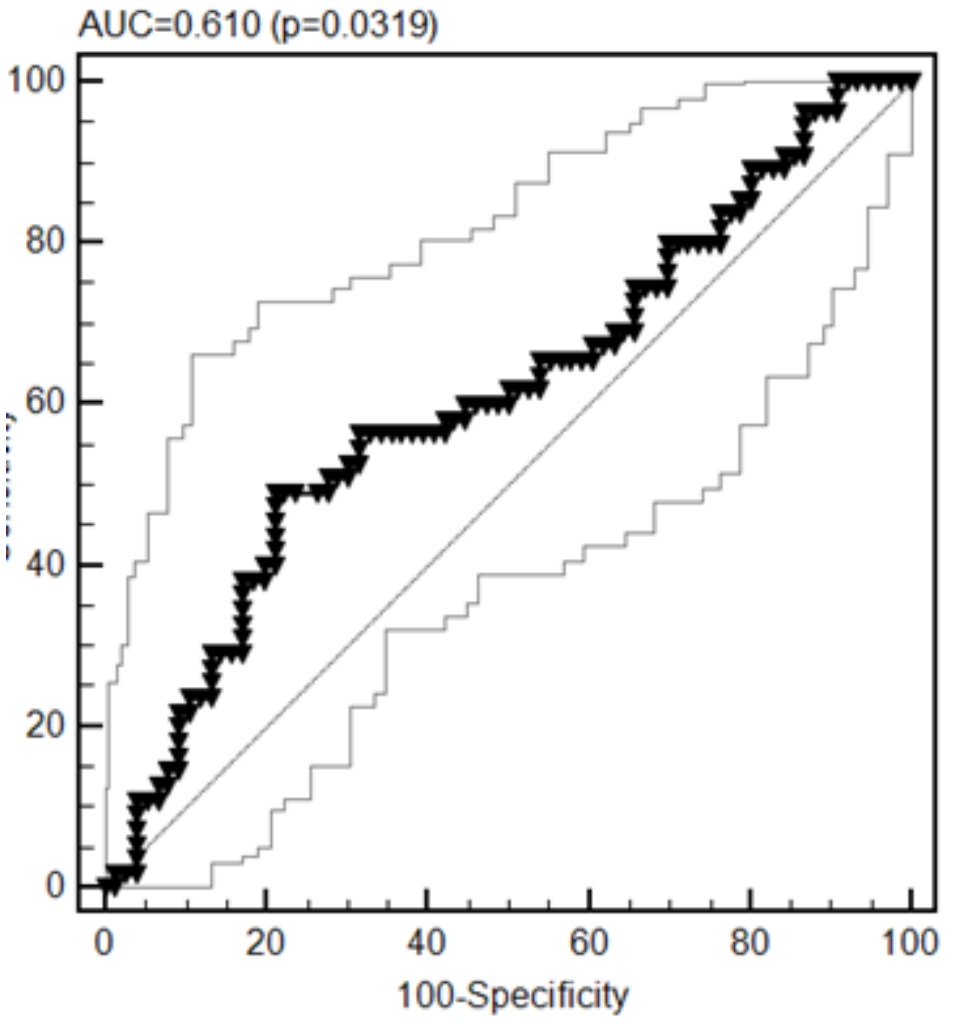

Figure 3 - Receiver operating characteristic curve for the systemic immune-inflammation index in predicting non-dippers. AUC: area under the receiver operating characteristic curve.

Increased inflammation may increase neutrophil and platelet counts while decreasing lymphocyte count. ${ }^{17}$ Inflammatory cytokines are secreted by monocytes as a result of inflammatory reactions, whereas HDL has anti-inflammatory effects. MHR has been identified as an easy cardiovascular prognostic marker indicating the intensity of inflammation. ${ }^{11}$
High levels of inflammatory markers were described in hypertensive patients with non-dipper BP. ${ }^{18,19}$ Inflammation may play a role in individuals being non-dipper, even if they are normotensive. Therefore, the patients' inflammatory conditions should be considered. Due to expensive and time-consuming procedures, the measurement of cytokines, adhesion molecules, and chemokines to assess 
inflammatory status is difficult. On the other hand, SII, MHR, PLR, and NLR are inflammation-based indexes that are cost-effective and can be easily obtained by routine blood tests. Based on this, we searched for an easy and accesible inflammation-based index to distinguish patients who may be non-dipper even if they are normotensive and who should be confirmed with an ABPM evaluation.

Previous studies have investigated the cardiovascular effects of a nocturnal decline in BP in normotensives, ${ }^{4,20}$ as well as in hypertensive patients. ${ }^{21,22}$ Cardiovascular mortality among dipper hypertensive patients and non-dipper normotensive patients is similar, ${ }^{23}$ and it is unknown if non dipper normotensives are candidates to being hypertensive. Therefore, diagnosing and managing non-dippers not only among hypertensives but also among normotensives is important. If their dipping status is not determined early enough, non-dipper normotensive individuals may not have the chance to receive an antihypertensive medication despite a higher cardiovascular risk. Hence, an SII cut-off may be useful to select patients who need to be evaluated with ABPM regarding their nocturnal BP pattern among those defined as normotensive according to office BP measurements.

Although in previous studies the association of inflammation with being non-dipper has been investigated in hypertensive patients, ${ }^{24,25}$ as far as we know, our study is the first to investigate the relationship between nocturnal BP pattern and inflammationbased indexes derived from complete blood count and biochemical test results in normotensive individuals.

\section{Limitations}

Firstly, we only searched for the relationship between dipping status and inflammation-based indexes derived from complete blood count and biochemical test results. Apart from these study parameters, we did not measure other inflammatory markers such as C-reactive protein or interleukin-6. Another limitation of our study is the lack of data on salt consumption on the day of measurement, the menopausal status of female participants, and sleep diaries including sleep-wake hours.

\section{Conclusion}

We observed a significant relationship between SII and a nocturnal decline of SBP in normotensives.
SII may be a predictive parameter for non-dipper normotensive individuals and may be useful to distinguish those who should be confirmed with ABPM in terms of dipping status before target organ damage develops. Further studies are needed on how to distinguish non-dipper individuals among normotensives both correctly and easily in daily practice.

\section{Acknowledgement}

From the bottom of my heart, I would like to express my special thanks to my father (M.Ugur Gunay), who has supported me throughout my life and has just passed away because of an incurable disease, unfortunately.

\section{Author contributions}

Conception and design of the research: Gunay $S$, Caliskan S. Acquisition of data: Caliskan S, Gunay S. Analysis and interpretation of the data: Gunay S. Sigirli D. Statistical analysis : Sigirli D. Writing of the manuscript: Gunay S. Critical revision of the manuscript for intellectual content : Gunay S.

\section{Potential Conflict of Interest}

No potential conflict of interest relevant to this article was reported.

\section{Sources of Funding}

There were no external funding sources for this study.

\section{Study Association}

This study is not associated with any thesis or dissertation work.

\section{Ethics approval and consent to participate}

This study was approved by the Ethics Committee of the Istanbul Bakirkoy Dr Sadi Konuk Training and Research Hospital under the protocol number 2019-1010. All the procedures in this study were in accordance with the 1975 Helsinki Declaration, updated in 2013. Informed consent was obtained from all participants included in the study. 


\section{References}

1. Fabbian F, Smolensky MH, Tiseo R, Pala M, Manfredini R, Portaluppi F. Dipper and Non-Dipper Blood Pressure 24-hour Patterns: Circadian Rhythm-Dependent Physiologic and Pathophysiologic Mechanisms. Chronobiol Int. 2013;30(1-2):17-30. doi: 10.3109/07420528.2012.715872.

2. Williams B, Mancia G, Spiering W, Agabiti Rosei E, Azizi M, Burnier M, et al. 2018 ESC/ESH Guidelines for the Management of Arterial Hypertension. Eur Heart J. 2018;39(33):3021-3104. doi: 10.1093/eurheartj/ ehy339.

3. Routledge FS, McFetridge-Durdle JA, Dean CR; Canadian Hypertension Society. Night-Time Blood Pressure Patterns and Target Organ Damage: A Review. Can J Cardiol. 2007;23(2):132-8. doi: 10.1016/s0828$282 x(07) 70733-x$.

4. Ohkubo T, Hozawa A, Yamaguchi J, Kikuya M, Ohmori K, Michimata M, et al. Prognostic Significance of the Nocturnal Decline in Blood Pressure in Individuals With and Without High 24-h Blood Pressure: The Ohasama Study. J Hypertens. 2002;20(11):2183-9. doi: 10.1097/00004872200211000-00017.

5. Polat M, Bugdayci G, Kaya H, Oğuzman H. Evaluation of Neutrophil-toLymphocyte Ratio and Platelet-to-Lymphocyte Ratio in Turkish Patients With Chronic Plaque Psoriasis. Acta Dermatovenerol Alp Pannonica Adriat. 2017;26(4):97-100. doi: 10.15570/actaapa.2017.28.

6. Sen BB, Rifaioglu EN, Ekiz O, Inan MU, Sen T, Sen N. Neutrophil to lymphocyte Ratio as a Measure of Systemic Inflammation in Psoriasis. Cutan Ocul Toxicol. 2014;33(3):223-7. doi: 10.3109/15569527.2013.834498.

7. Yurtdaş M, Yaylali YT, Kaya Y, Ozdemir M, Ozkan I, Aladağ N. Neutrophil-to-Lymphocyte Ratio May Predict Subclinical Atherosclerosis in Patients With Psoriasis. Echocardiography. 2014;31(9):1095-104. doi: 10.1111/echo.12511.

8. Ganjali S, Gotto AM Jr, Ruscica M, Atkin SL, Butler AE, Banach M, et al. Monocyte-to-HDL-Cholesterol Ratio as a Prognostic Marker in Cardiovascular Diseases. J Cell Physiol. 2018;233(12):9237-9246. doi: $10.1002 /$ jcp.27028.

9. Jomrich G, Gruber ES, Winkler D, Hollenstein M, Gnant M, Sahora K, et al. Systemic Immune-Inflammation Index (SII) Predicts Poor Survival in Pancreatic Cancer Patients Undergoing Resection. J Gastrointest Surg. 2020;24(3):610-618. doi: 10.1007/s11605-019-04187-z.

10. Turkmen D, Altunisik N, Sener S. Investigation of Monocyte HDL Ratio as an Indicator of Inflammation and Complete Blood Count Parameters in Patients With Acne Vulgaris. Int J Clin Pract. 2020;74(12):e13639. doi: 10.1111/ijcp.13639.

11. Canpolat U, Çetin EH, Cetin S, Aydin S, Akboga MK, Yayla C, et al. Association of Monocyte-to-HDL Cholesterol Ratio with Slow Coronary Flow is Linked to Systemic Inflammation. Clin Appl Thromb Hemost. 2016;22(5):476-82. doi: 10.1177/1076029615594002.

12. Cakır I, Arifoğlu H, Günay N, Pangal E, Şahin D, Sert AG, et al. Monocyte to High Density Lipoprotein Ratio: A Novel Inflammation Marker Related With Diabetic Retinopathy. Erciyes Med J. 2020;42(2):190-4. doi: 10.14744/etd.2020.32549.
13. Kaya MG, Yarlioglues M, Gunebakmaz O, Gunturk E, Inanc T, Dogan A, et al. Platelet Activation and Inflammatory Response in Patients With Non-Dipper Hypertension. Atherosclerosis. 2010;209(1):278-82. doi: 10.1016/j.atherosclerosis.2009.09.010.

14. Gunebakmaz O, Kaya MG, Duran M, Akpek M, Elcik D, Eryol NK Red Blood Cell Distribution Width in 'Non-Dippers' versus 'Dippers'. Cardiology. 2012;123(3):154-9. doi: 10.1159/000342667.

15. Yılmaz M, Kayançiçek H. A New Inflammatory Marker: Elevated Monocyte to HDL Cholesterol Ratio Associated with Smoking. J Clin Med. 2018;7(4):76. doi: 10.3390/jcm7040076.

16. Zhang J, Zhang HY, Li J, Shao XY, Zhang CX. The Elevated NLR, PLR and PLT May Predict the Prognosis of Patients With Colorectal Cancer: A Systematic Review and Meta-Analysis. Oncotarget. 2017;8(40):6883768846. doi: 10.18632/oncotarget.18575.

17. Zhang Y, Yin Y, Kuai S, Shan Z, Pei H, Wang J. Combination of Neutrophil to Lymphocyte Ratio and Platelet to Lymphocyte Ratio as Diagnostic Biomarker for Rheumatoid arThritis. Int J Clin Exp Med. 2016;9(11):22076-81.

18. von Känel R, Jain S, Mills PJ, Nelesen RA, Adler KA, Hong S, et al. Relation of Nocturnal Blood Pressure Dipping to Cellular Adhesion, Inflammation and Hemostasis. J Hypertens. 2004;22(11):2087-93. doi: 10.1097/00004872-200411000-00009.

19. Turak O, Ozcan F, Tok D, Işleyen A, Sökmen E, Taşoğlu I, et al. Serum Uric Acid, Inflammation, and Nondipping Circadian Pattern in Essential Hypertension. J Clin Hypertens. 2013;15(1):7-13. doi: 10.1111/jch.12026.

20. Soylu A, Duzenli MA, Yazici M, Ozdemir K, Tokac M, Gok H. The Effect of Nondipping Blood Pressure Patterns on Cardiac Structural Changes and left Ventricular Diastolic Functions in Normotensives. Echocardiography. 2009;26(4):378-87. doi: 10.1111/j.1540-8175.2008.00821.x.

21. Fagard RH, Thijs L, Staessen JA, Clement DL, De Buyzere ML, De Bacquer DA. Night-Day Blood Pressure Ratio and Dipping Pattern as Predictors of Death and Cardiovascular Events in Hypertension. J Hum Hypertens. 2009;23(10):645-53. doi: 10.1038/jhh.2009.9.

22. Verdecchia P, Schillaci G, Reboldi G, Franklin SS, Porcellati C. Different Prognostic Impact of 24-hour Mean Blood Pressure and Pulse Pressure on Stroke and Coronary Artery Disease in Essential Hypertension. Circulation. 2001;103(21):2579-84. doi: 10.1161/01.cir.103.21.2579.

23. Hermida RC, Ayala DE, Mojón A, Fernández JR. Blunted SleepTime Relative Blood Pressure Decline Increases Cardiovascular Risk Independent of Blood Pressure Level--the "Normotensive Non-Dipper" Paradox. Chronobiol Int. 2013;30(1-2):87-98. doi: 10.3109/07420528.2012.701127.

24. Stefanadi E, Tousoulis D, Androulakis ES, Papageorgiou N, Charakida M, Siasos G, et al. Inflammatory Markers in Essential Hypertension: Potential Clinical Implications. Curr Vasc Pharmacol. 2010;8(4):509-16. doi: 10.2174/157016110791330870.

25. Harrison DG, Guzik TJ, Lob HE, Madhur MS, Marvar PJ, Thabet SR, et al. Inflammation, Immunity, and Hypertension. Hypertension. 2011;57(2):132-40. doi: 10.1161/HYPERTENSIONAHA.110.163576. 